\title{
Kernos
}

Revue internationale et pluridisciplinaire de religion grecque antique

20 | 2007

Varia

\section{Généalogie et historiographie : une réécriture de la généalogie des rois de Sparte}

Le cas de Démarate chez Hérodote

\section{Gerasimoula Zographou}

\section{(2) OpenEdition}

Journals

Édition électronique

URL : https://journals.openedition.org/kernos/412

DOI : $10.4000 /$ kernos.412

ISSN : 2034-7871

Éditeur

Centre international d'étude de la religion grecque antique

Édition imprimée

Date de publication : 1 janvier 2007

Pagination : 189-204

ISSN : 0776-3824

Référence électronique

Gerasimoula Zographou, «Généalogie et historiographie : une réécriture de la généalogie des rois de Sparte », Kernos [En ligne], 20 | 2007, mis en ligne le 24 mai 2011, consulté le 08 septembre 2022. URL : http://journals.openedition.org/kernos/412 ; DOI : https://doi.org/10.4000/kernos.412 


\title{
Généalogie et historiographie : une réécriture de la généalogie des rois de Sparte. Le cas de Démarate chez Hérodote*
}

\begin{abstract}
Résumé : Afin d'expliquer l'hostilité des deux rois de Sparte Démarate et Cléomène, remontant à leurs ancêtres, Hérodote fait une digression concernant leur généalogie, leurs privilèges ainsi que la généalogie de Démarate. Cette digression permet l'exploitation de la généalogie tant sur le plan politique que sur le plan narratif. Au premier plan apparaît d'une manière sous-jacente l'idée que Démarate possède un droit héréditaire sur le trône supérieur à celui de Cléomène, à cause de son rapport aux éléments surnaturels présents dans sa généalogie : a) à travers l'intervention d'Hélène, qui a provoqué l'amour d'Ariston, roi de Sparte, pour la mère de Démarate; b) à travers une double paternité, parce qu'elle ignorait s'il était fils d'Ariston, un mortel, ou du héros Astrabakos. Ainsi, en la personne de Démarate convergent les deux pouvoirs, religieux et politique. L'ambiguité de cette généalogie a été exploitée par ses ennemis afin d'obtenir son expulsion. Sur le plan narratif, Hérodote exploite la généalogie de Démarate pour le présenter comme une victime d'une intrigue impie. L'historien justifie l'action de Démarate sur le plan politique, tandis que sur le plan moral celle-ci a été déjà justifiée par le tíøıৎ que, selon Hérodote, ses adversaires ont payé par leur mort atroce.
\end{abstract}

Abstract: In his effort to explain the hostility between the two Spartan kings Demaratus and Kleomenes, which dated back to their ancestors, Herodotus makes a digression concerning their genealogy, their privileges and the genealogy of Demaratus. From this digression, an exploitation of the genealogy emerges both on the political and the narrative level. On the first level, it is implied that Demaratus has a hereditary right to the throne, which is greater than that of Kleomenes, because of his relationship with the supernatural element. This supernatural element appears in his genealogy a) through the intervention of Helen, a fact which brought about the love of Ariston, king of Sparta, for the mother of Demaratus; b) through a double paternity, since she ignored the fact that he was the offspring of either Ariston, a mortal, or of the hero Astrabakus. In this way, in the figure of Demaratus, the two capacities, the religious and the political, converge. His enemies exploit the ambiguity of this genealogy in order to achieve his dethronement. On the narrative level, Herodotus exploits the genealogy of Demaratus in order to present him as a victim of an impious conspiracy. Therefore, he justifies him politically, while, morally he has already been justified by tisis for which, according to Herodotus, his adversaries paid by their atrocious death.

\footnotetext{
* Cet article est issu d'une communication présentée à la Table Ronde du S.I.R.E., au colloque du C.I.E.R.G.A. (Bruxelles, Septembre 2005, voir Kernos 2006). Je remercie L. Couloubaritsis, I. Papadopoulou et V. Pirenne-Delforge de m'avoir invitée et d'avoir relu et amélioré la version française du texte. Je remercie également mes collègues I.N. Perysinakis, A. Tatti, S. Constantinidou, E. Gasti, A. Zographou, qui ont lu des versions précédentes de l'essai et m'ont fait de précieuses remarques.
} 


\section{Introduction}

Dans une des digressions qui abondent dans son œuvre, Hérodote décrit la situation des villes les plus puissantes de la Grèce à la veille de la première campagne perse. Il s'agit de sa contribution la plus importante à l'histoire des constitutions grecques ${ }^{1}$. En cette période, Sparte se trouvait au summum de l'hostilité entre ses deux rois, Démarate et Cléomène (VI, 51). Le sujet de la digression est la crise de l'institution politique souveraine de la cité, qui s'inscrit sur deux plans : le premier concerne le rapport personnel entre les deux rois², tandis que le deuxième met en scène le statut des deux maisons royales, ce qui inclut la nouvelle présentation inattendue de Démarate $(c f$. V, 75). Les deux concurrents proviennent du même arbre généalogique ${ }^{3}$, mais non de la même maison royale. Démarate appartient à une maison inférieure à celle de Cléomène qui ne se différencie pourtant que par l'aînesse de son ancêtre Eurysthénès, le chef de la maison des Agiades, tandis que le chef de l'autre maison royale, celle des Eurypontides, était Proklès. Dans le texte hérodotéen transparaît une volonté de défendre l'égalité des deux maisons, égalité que certains Lacédémoniens contestent en promouvant l'idée que la tension dans les relations entre les deux hommes, en dehors des différences personnelles, reflète aussi l'hostilité familiale; celle-ci remonterait à leur origine et aurait une influence sur le fonctionnement de l'institution royale à Sparte.

Ainsi, parallèlement à la description de la vie politique de Sparte à la veille du conflit gréco-perse, Hérodote entreprend d'interroger la discorde entre les deux agents politiques souverains. Nous verrons que leurs généalogies constituent l'élément prédominant sur les plans personnel et familial, tant dans le passé que dans le présent, et c'est précisément cet aspect qui intéresse notre enquête. En fait, notre intention n'est pas de nous pencher sur la question de l'arbre généalogique des rois de Sparte, qui a préoccupé à maintes reprises les spécialistes ${ }^{4}$, mais d'examiner comment la généalogie a fonctionné dans la vie

${ }^{1}$ Voir W.W. How et J. WeLtS, A Commentary on Herodotus with Introduction and Appendixes, Vol. II (Books V-IX), Oxford, 1980 [19282], aux ch. 51-60.

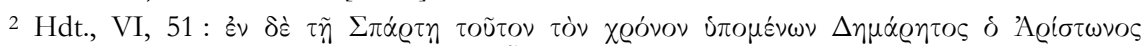

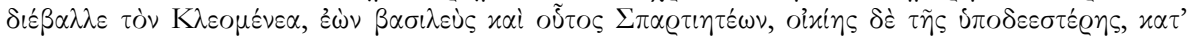

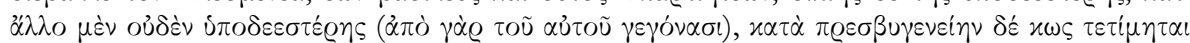

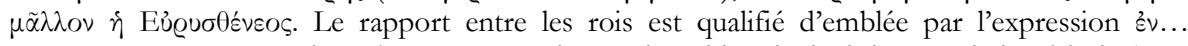

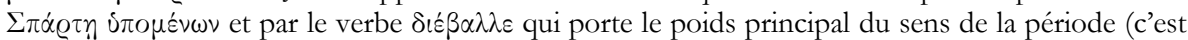
pourquoi il est placé en avant en perturbant la syntaxe). Figure d'inversion (= i $\pi \varepsilon Q \beta \alpha \tau o ́ v)$ à la

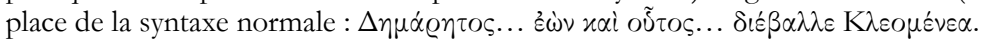

3 Voir E.I. MCQueEn, Herodotus Book VI. Edited with Introduction and Commentary, London, 2000, ad loc. 51.

${ }^{4}$ La liste qu'Hérodote mentionne dans la suite concerne les rois de Sparte après le retour des Héraclides jusqu'à la séparation de leur arbre généalogique pendant l'époque d'Aristodémos. Il existe aussi d'autres listes représentatives des deux maisons royales qui en ont résulté, les listes des Agiades et des Eurypontides, qui font surgir de nombreuses interrogations sur lesquelles on 
politique de Sparte, et comment Hérodote l'a exploitée dans son œuvre. L'hostilité personnelle et familiale couvrent, dans l'ordre inverse, la première (VI, 51-55) et la dernière (VI, 61-70) partie du récit, tandis que la partie intermédiaire (VI, 56-60) décrit les privilèges des rois spartiates. Comme cette partie n'est pas en relation directe avec notre étude, nous ne porterons notre attention que sur les rapports entre généalogie, hostilité familiale et hostilité individuelle. Nous suivrons le fonctionnement des généalogies simultanément aux niveaux politique et narratif, car les deux niveaux sont intimement liés.

\section{Généalogie et hostilité familiale}

Pour construire son argumentation en faveur de l'égalité des deux maisons royales, Hérodote remonte, par la technique du retardement $\left(\mu \varepsilon \dot{\lambda} \lambda \eta \eta \iota^{5}\right)$ via une analepse interrompant la narration ${ }^{6}$, à la 'préhistoire' de la famille, puisée dans le témoignage des Lacédémoniens (VI, 52, 1-8). Au mythe des jumeaux, récit fantastique sur la double royauté, Hérodote utilise l'oratio obliqua en contestant implicitement sa fiabilité

Comme il résulte de l'introduction, les Lacédémoniens s'attachent d'abord à mettre en valeur la généalogie d'Aristodémos en le faisant remonter directement à Héraclès via son fils Hyllos afin de renforcer leur souveraineté sur la région, où ils sont jadis arrivés en tant que colons 8 ; ils s'efforcent ensuite de convaincre que c'est le roi lui-même qui les a amenés à Sparte et non ses enfants. C'est pour cette raison, semble-t-il, qu'ils refusent la version poétique, qui annule le rôle régulateur d'Aristodémos concernant les évolutions à Sparte, puisqu'elle

peut voir : D.W. PrakKEN, « Herodotus and the Spartan King Lists », TAPhA 71 (1940), p. 460472; C.G. STARR, "The Credibility of Early Spartan History », Historia 14.3 (1965), p. 268-269; K.M. Cragg, Herodotus' Presentation of Sparta, Diss., Michigan, 1976, p. 29; P. CarTLEDGE, Sparta and Laconia. A Regional History 1300-362 BC., London, 1979, Appx. 3, p. 341-346; C. Calame, "Le récit généalogique spartiate : la répresentation mythologique d'une organization spatiale", QS 25-26 (1987), p. 41-81; J.-Cl. CARRIÈRE, " Du mythe à l'histoire. Généalogies héroïques, chronologies légendaires et historicisation des mythes», in D. AUGER et S. SAÏD (éds), Généalogies Mythiques. Actes du VIIIe Colloque du Centre de Recherches Mythologiques de l'Université de Paris-X (Chantilly, 14-16 septembre 1995), Paris, 1998, p. 68 sq.; L. SCOTT, Historical Commentary on Herodotus Book 6, Leiden, Boston, 2005, p. 263-264.

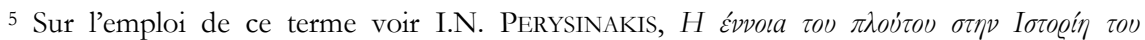

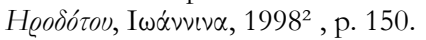

${ }^{6}$ Sur l'usage des termes 'retardement', 'analepse' et sur les autres termes concernant le style voir I.J.F. DE JONG, «Aspects narratologiques des Histoires d'Hérodote», Lalies 19 (1999), p. 242 sq.; cf. A. Rengakos, «Epic Narrative Technique in Herodotus' Histories », SemRom 4.2 (2001), p. 253-270.

7 Sur le fonctionnement de l'oratio obliqua chez Hérodote voir G.L. COOPER, «Intrusive oblique infinitives in Herodotus », TAPhA 104 (1974), p. 23-76; SCOTT, o.c. (n. 4), p. 23.

8 Nous reconnaissons ici le thème des prétentions territoriales qui sont fondées sur des parentés avec des dieux et des héros. Voir par ex. R.W. MACAN, Herodotus. The Fourth, Fifth, and Sixth Books. With Introduction, Notes, Appendices, Indices, Maps, Vols I-II, London/New York, 1973 [1895], ad loc. 51, 3; CARTLED GE, o.c. (n. 4), p. 343. Cf. CALAME, l.c. (n. 4), p. 51 sq. 
présuppose son décès avant l'invasion des Doriens dans le Péloponnèse9. C'est exactement ce rôle $\left(\beta a \sigma \lambda \varepsilon_{\varepsilon} \omega \omega v\right)$ qui répond aux objections des poètes et signale la deuxième des conditions qui appuient les droits héréditaires des ses enfants au trône, la première étant la relation généalogique avec le divin.

Selon la version des Lacédémoniens, peu après leur arrivée à Sparte sous le

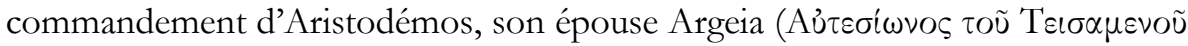

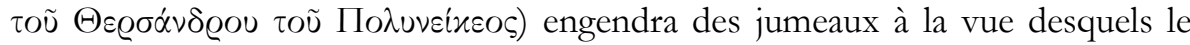
roi tomba malade et mourut. Cet accouchement gémellaire constitue un élément structurel du récit, qui opère sur plusieurs plans : la généalogie, la relation interpersonnelle entre Eurysténès et Proclès, qui crée un problème au niveau de la réalité politique, problème qui s'étend au niveau symbolique. Ce dernier se construit par une mise en relation des fils jumeaux d'Aristodémos et des jumeaux Castor et Pollux, les chefs/ prêtres / rois mythiques de Sparte ${ }^{10}$. La narration vise ainsi à renforcer le lien entre les deux rois de Sparte et les Dioscures qui assurent à l'institution de la double royauté la garantie divine.

Sur le plan de la réalité politique, l'accouchement gémellaire a mis en marche les événements conduisant à l'imposition de la double royauté, en créant une confusion concernant l'âge des enfants, facteur déterminant de la succession. La question critique est de pouvoir réconcilier l'égalité avec la priorité. Le père est mort avant d'avoir pu nommer son successeur; les Lacédémoniens sont dans l'impossibilité de choisir le roi sur base des qualités naturelles des deux enfants. La mère, qui tient la clé de l'évolution politique, refuse de collaborer, avec l'arrière-pensée de proclamer rois ses deux enfants, orientant les choses selon sa volonté11. Comme les moyens humains pour résoudre le problème semblent épuisés, on s'adresse à l'oracle, qui entretient la confusion par un conseil

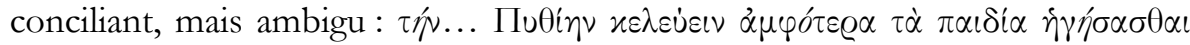

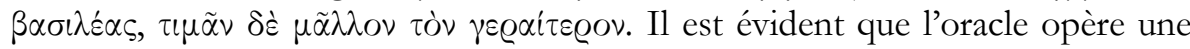
manœuvre diplomatique pour concilier la situation avérée et la loi spartiate qui prescrit de proclamer roi l'aîné. La position de l'oracle favorise la double royauté et l'un des jumeaux ${ }^{12}$. Le problème se résout seulement en partie, tandis

\footnotetext{
9 Pausanias III, 1, 5-6; Apollodore II, 8, 2, avec les détails sur le décès d'Aristodémos. Dans leurs descriptions, les spécialistes reconnaissent la version poétique. Voir BAHR, selon MACAN, supra, ad loc. 52, 2; G. NENCI, Erodoto, Le storie, Volume VI, LibroVI:La battaglia di Maratona, Fondazione Lorenzo Valla, 1998, ad loc. 52, 1-4; MCQUEEN, o.c. (n. 3), ad loc. 52, 1.

10 Voir B. Sergent, «Le partage du Péloponnèse entre les Héraklides », RHR 192 (1977), p. 128 et 133. Sur la relation des jumeaux avec les Dioscures voir P. CARLIER, La royauté en Grèce avant Alexandre, Strasbourg, 1984, p. 298-301. Cf. E. Millender, "Herodotus and Spartan Despotism », in A. Powell \& S. Hodkinson (éds), Sparta Beyond the Mirage, London, 2002, p. 12; E. LÉVY, Sparte. Histoire politique et sociale jusqu'à la conquête romaine, Paris, 2003, p. 164.

11 Sur la contribution déterminante d'Argeia à l'établissement de la double royauté voir E. MiLlender, «Athenian Ideology and the Empowered Spartan Woman », in S. HodKINSON \& A. Powell (éds), Sparta. New Perspectives, London, 1999, p. 357.

12 Sur la relation de l'oracle de Delphes avec Sparte depuis le début de son développement et sur son rôle dans la prise des décisions voir H.W. PARKE, D.E.W. WORMELL, The Delphic Oracle,
} 
que la confusion qui découle de l'ambiguité de l'oracle se retourne sous une autre forme: que signifie exactement le verbe $\tau \iota \mu \tilde{\alpha} \nu$ ?13 Et, surtout, qui est

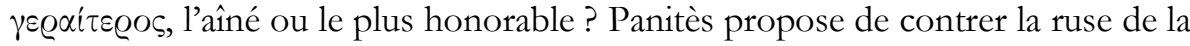
mère en surveillant l'ordre des soins de ses enfants pour comprendre qui est

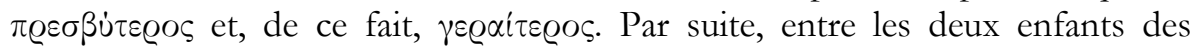
mêmes parents, nés íøoı et öroı par gestation jumelle, on choisit Eurysthénès sur base d'un critère incertain : le plus âgé doit être celui que la mère soigne le premier, ce qui, selon les affirmations des Lacédémoniens (VI, 52, 8), créa une hostilité permanente entre les deux frères et leurs descendants.

Finalement, la conjoncture pousse à l'imposition de la double royauté, c'està-dire à l'institutionnalisation du dilemme via une ambiguitté réitérée: les enfants sont jumeaux, la présence d'Aristodémos est incertaine, Argeia connait la vérité mais simule l'ignorance, l'oracle est ambigu. Le récit suggérerait-il l'idée que certains ont tiré parti de l'ambiguité pour revendiquer des privilèges, grâce à la médiation de l'oracle ? Dans ce cas, c'est peut-être Eurysthénès lui-même ou ses descendants qui ont été favorisés. Ailleurs, dans les Histoires on trouve d'autres exemples d'implication de l'oracle dans des jeux politiques ${ }^{14}$.

L'analyse précédente a montré que le récit considéré avait deux objectifs. Il s'agissait tout d'abord de mettre en évidence la généalogie des chefs de l'invasion dorienne, afin de fonder leurs droits au pouvoir et de légitimer le partage de la région entre les colons Héraclides. Il s'agissait ensuite d'intégrer et d'interpréter l'établissement de la double royauté à Sparte ${ }^{15}$, ainsi que de justifier le traitement spécial dont jouissaient Eurysthénès et ses descendants, à l'origine de l'hostilité entre Démarate et Cléomène. Le récit a comme objectif ultime de légitimer la situation politique de Sparte. En d'autres termes, la généalogie est chaque fois exploitée pour confirmer des privilèges et c'est pour cette raison qu'on constate des frictions entre les membres des deux maisons royales.

Par conséquent, l'étude de la version spartiate montre comment une histoire pittoresque, pleine de coïncidences étranges et d'intrigues a pu se fonder sur une généalogie, pour servir la propagande politique de l'époque; autrement dit,

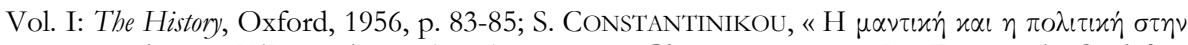

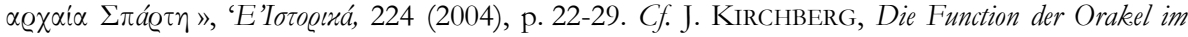
Werke Herodots, Hypomnemata 11, Göttingen, 1965, p. 59-70.

13 Sur les interprétations du verbe dans ce contexte voir MACAN, o.c. (n. 8), ad loc. 52, 18; R. Crahay, La littérature oraculaire chez Hérodote, Paris, 1956, p. 160; CARLIER, o.c. (n. 10), p. 288.

${ }^{14}$ Voir par ex. la subornation de l'oracle par les Alcméonides afin d'arracher une réponse politique qui leur soit favorable (Hdt. V, 63; cf. V, 66).

${ }^{15} \mathrm{La}$ corrélation entre le récit et les événements politiques à Sparte a déjà été faite par d'autres spécialistes, mais dans le but de rechercher à travers le récit les données historiques de l'époque. Sur les conjectures diverses, voir à titre indicatif: MACAN, o.c. (n. 8), ad loc. 51, 3; How Wells, o.c. (n. 1), p. 82-83; MCQueEn, o.c. (n. 3), ad loc. 51; W.G. Forrest, A History of Sparta 950-192 B.C., London, 1968, p. 28-29; SERGENT, l.c. (n. 10), p. 163 et « La présentation spartiate de la royauté », RHR 189 (1976), p. 3-52; LÉVY, o.c. (n. 10), p. 163; SCOTT, o.c. (n. 4), ad loc. 51. 
elle montre comment le procédé narratif sert le politique ${ }^{16}$. Une mise en rapport similaire du procédé narratif et du plan politique sert aussi les intentions d'Hérodote. Sur le plan narratif, la généalogie permet l'exploration et l'exploitation des domaines vagues et obscurs pour les humains, tels que la relation avec le divin ou avec des femmes, domaines difficiles à contrôler même par des hommes puissants, tels qu'Ariston et Démarate. Nous reconnaissons ici des figures et des idées familières à l'œuvre hérodotéenne. Ariston est mort jeune apparemment en compensation des faveurs que le sort lui a accordées (comme Cléobis et Biton) ${ }^{17}$ : être roi, marié à une femme illustre et avoir eu deux enfants; la mère intelligente qui manipule les autorités jouant un rôle important dans les évolutions politiques (comme la mère de Démarate, Labda, Atossa, Phérétimé); Panitès, «le vieil homme sage qui se met en avant pour résoudre le problème $»^{18}$. Sur le plan politique, la généalogie sert à la défense de la thèse de l'historien selon laquelle Démarate, ayant les mêmes droits au pouvoir que Cléomène, n'avait pas de raisons de le haïr. L'inégalité, s'il y en a eu une, découle du respect dû à celui qui a été désigné, par une procédure hasardeuse, comme le chef le plus âgé de la maison royale. Nous découvrons ici Hérodote le chercheur, qui mentionne ses sources, les évalue et les oppose à d'autres, qui structure son discours de façon démonstrative, intervient, lorsqu'il le juge indispensable, et qui critique sa matière, si nécessaire.

La version suivante ${ }^{19}$, celle des Grecs, a la préférence d'Hérodote parce qu'elle lui permet de révéler, surtout, et de revaloriser l'origine commune de Démarate et de Cléomène en la rapportant directement à l'être divin suprême, Zeus ( $c f$. VII, 61, 3). Il s'agit de renforcer l'identité hellénique des rois de Sparte $(V I, 53,2)$ et d'insister sur l'ancienneté de la royauté spartiate. En associant l'arbre généalogique des rois de Sparte à l'Égypte, il touche ainsi un de ses sujets de prédilection, la relation de la culture grecque avec la culture égyptienne. Dans ce même but, prenant l'arbre généalogique comme véhicule et comme outil pour la datation ${ }^{20}$, il s'étend aussi vers le passé mythique, tandis que les Spartiates arrêtent l'énumération des générations royales à l'époque 'historique'

${ }^{16}$ Voir J.T. HoOKer, «Spartan Propaganda », in A. POWELL (éd.), Classical Sparta: Techniques bebind her Success, London, 1989, p. 122.

17 Th. Harrison, Divinity and History. The Religion of Herodotus, Oxford, 2000, p. 53: «Good fortune signals impending misfortune. »

18 Voir SCOTT, o.c. (n. 4), ad loc. 52, 5. Cf. Crahay, o.c. (n. 13), p. 160.

${ }_{19}$ Pour une analyse plus détaillée de cette version et de celle des Perses, voir G. ZoGRAPHOU, «Histoire et narrativité : la généalogie de Démarate selon Hérodote » à paraître dans la revue

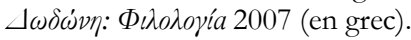

20 Voir par ex. F. Mitchel, "Herodotos' Use of Genealogical Chronology », Phoenix 10 (1956), p. 48-69; CARRIÈRE, l.c. (n. 4), p. 68 sq. Cf. C. DARBO-PESCHANSKI, Le discours du particulier. Essai sur l'enquête hérodotéenne, Paris, 1987, p. 25 sq. 
de l'invasion dorienne sous les Héraclides ${ }^{21}$ - ce dernier élément marque la différence des deux versions.

Tout le récit précédent constitue une procédure de démonstration qui vise à prouver que l'origine de Démarate est aussi illustre que celle de son rival. Hérodote exploite ici la généalogie pour de nombreuses raisons : structurer sa narration selon un ordre logique et causal en remontant aux origines du conflit des deux rois, créer des types de comportement et faire paraitre ses idées, notamment pour soutenir sa thèse politique d'après laquelle les deux maisons royales sont essentiellement équivalentes et ont une origine illustre commune.

\section{Généalogie et hostilité individuelle}

La fin de la digression consacrée aux histoires de famille relatives à la double royauté et les privilèges des rois de Sparte ${ }^{22}$ présente la structure d'une «ring composition ${ }^{23}$, brève mais essentielle, par rapport à l'évolution de l'action, car Hérodote compare les stratégies des deux hommes afin que la conduite de Démarate apparaisse encore plus intéressée et blâmable en comparaison avec l'action désintéressée de son rival pour le bien de la Grèce ${ }^{24}$. Bien plus, il éclaire la cause du conflit en attribuant à Cléomène une action politique dans le cadre de la politique extérieure de Sparte (expliquant ainsi le sens du participe

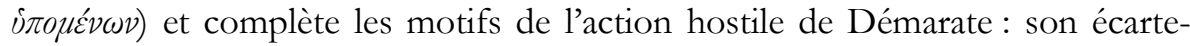
ment et les sentiments consécutifs de jalousie et d'envie, fait qui a conduit Hérodote à défendre Cléomène, contrairement à son habitude ${ }^{25}$. Ce dernier, après son retour d'Égine, a décidé de venger son corrégnant tirant prétexte de sa généalogie. Sa vengeance et les conséquences qu'elle a entraînées sont

\footnotetext{
${ }^{21}$ Sur la limitation par Hérodote de l'époque mythique, à laquelle Persée appartient, ainsi que sur le 'Retour des Héraclides' en tant que « la limite qui sépare pour les Grecs l'époque héroïque et le temps historique », voir CARRIÈRE, l.c. (n. 4), p. 68 et 52.

22 Sur ce dernier sujet ayant pour but, entre autres, nous semble-t-il, de servir la logique de l'égalité des deux rois, ainsi que de faire allusion à leur rôle dans l'hostilité individuelle, voir A.H.M. JONES, Sparta, Oxford, 1967, p. 13-15; LÉVY, o.c. (n. 10), p. 167. Cf. SCOTT, o.c. (n. 4), p. $230-252$.

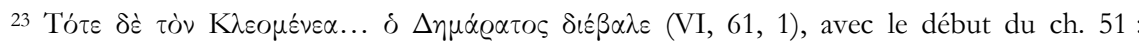

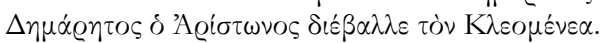

${ }^{24}$ Sur la contribution importante de Cléomène à l’affaire de l'Égine voir G.E.M. DE STE Croix, "Herodotus and King Cleomenes I of Sparta », in D. HARvey \& R. PARKER (éds), Athenian Democratic Origins and other essays, Oxford/New York, 2004, p. 428; sur le comportement contradictoire d'Hérodote envers Démarate voir A. Dovatour, "La menace de Démarate ", REG 50 (1937). Cf. D. BoedeKER, "The Two Faces of Demaratus", in D. BoEdeker, I. PERADOTTO (éds), Herodotus and the Invention of History, Arethusa 20 (1987), p. 185-201.

25 Voir par ex. V, 39; 42; 51; VI, 74-75. Sur l'attitude contradictoire d'Hérodote envers Cléomène voir Th. LENSCHAU, «König Kleomenes I von Sparta », Klio 31 (1938), p. 412; G.L. Huxley, Early Sparta, London, 1962, p. 77; E.N. Tigerstedt, The Legend of Sparta in Classical Antiquity, Lund, 1965, p. 90-91; CraGg, o.c. (n. 4), p. 39; DE STE Croix, supra, p. 422. Cf. J. HART, Herodotus and Greek. History, London/Canberra/New York, 1982, p. 124, 134 sq.; CARTLEDGE, o.c. (n. 4), p. 124.
} 
décrites dans deux grandes unités, qui nous occuperont par la suite : la première (1: VI, 61-66) concerne les événements qui ont provoqué son détrônement, la deuxième (2:VI, 67-70) ceux qui l'ont obligé de se réfugier en Perse.

\subsection{Le cheminement de Démarate jusqu'à son éviction}

Dans ce récit on discerne trois subdivisions qui se réfèrent à son origine [(1) VI, 61.2-63], à l'exercice de la royauté [(2) VI, 64] et au détrônement [(3) VI, 65-66], c'est-à-dire qu'elles traitent le prétexte, la cause de la vengeance ainsi que la manière dont elle a été réalisée.

\subsubsection{Origine (version commune) et accès au pouvoir}

Hérodote nous informe d'abord sur le père de Démarate : il s'est marié deux fois sans avoir d'enfants et, ignorant sa propre responsabilité dans ce mariage infécond, il a contracté un troisième mariage. Notons deux éléments qui déterminent la suite du récit: Ariston était roi lorsque Démarate est né, ce qui garantit ses droits au trône; d'autre part, il était incapable de procréer, fait qui annonce d'avance une paternité douteuse. Les enchaînements, qui rappellent le cas de Cléomène, sont inévitables : son père, lui aussi roi de Sparte, ne pouvait pas non plus avoir d'enfants de sa légitime épouse, sans en être responsable. Il a aussi contracté un deuxième mariage, duquel il a eu un fils, Cléomène. La différence entre les deux cas réside sur le fait que l'origine de ce dernier n'est pas douteuse même si le statut de sa mère peut paraitre problématique ${ }^{26}$.

De la mère de Démarate, nous ne connaissons que son origine noble et l'histoire de sa transformation miraculeuse de petite fille laide en la femme la plus belle de Sparte, suite à l'intervention d'Hélène répondant aux prières de sa nourrice ${ }^{27}$. Cette intervention divine opère à deux niveaux : au niveau symbolique, elle manifeste la faveur de la déesse envers la mère de Démarate indiquant une relation privilégiée de celui-ci avec le divin; au niveau de l'action, cette intention a provoqué la transformation de la femme et, à travers celle-ci, l'amour d'Ariston pour elle. Celui-ci a pu l'arracher à son conjoint au moyen d'un subterfuge, en l'occurrence un serment trompeur qui, à cause de son caractère inviolable, a obligé Agétos à lui céder sa femme, contribuant au renversement de la situation politique.

Une série d'événements, résultant tant de facteurs surnaturels que de l'initiative et de la responsabilité humaines, s'enchaînent en entraînant des conséquences inévitables : la stérilité d'Ariston a conduit au troisième mariage dicté par son grand amour pour la mère de Démarate, qui lui a été cédée de force par son ex-conjoint. L'amour étant le levier des évolutions, le rôle d'Hélène semble déterminant au niveau symbolique également. Sa présence est diffuse dans le récit, alors que se profilent de nombreuses ressemblances avec son mythe: la

\footnotetext{
26 Voir LÉVY, o.c. (n. 10), p. 165.

${ }^{27}$ Cf. Harrison, o.c. (n. 17), p. 80.
} 
mère de Démarate apparaît dans un rôle analogue au sien, alors que deux hommes, Agétos et Ariston la revendiquent. Le dernier rappelle Pâris, qui, amoureux de la belle Tyndaride, l'arrache à son mari de manière déshonorante. Plus tard nous verrons Démarate lui-même dans un rôle similaire. Même le thème de la double paternité est présent dans le mythe d'Hélène. Il n'y manque même pas l'élément du serment, qui renvoie aux serments des fiancés de celleci, même si son contenu est différent dans le cas d'Ariston ${ }^{28}$. La synergie de la faveur divine et du désir humain, personnel mais aussi collectif, aurait conduit à la naissance de Démarate. C'est ce que semble croire Hérodote, qui entreprend d'associer étymologiquement son nom au vœu de tous les Spartiates souhaitant que le meilleur roi qu'ils ont eu puisse avoir un fils ${ }^{29}$. Ariston, troublé au moment de la naissance de son fils, le désavoua un court instant devant les éphores. Action irréfléchie, dont Démarate paya les conséquences, quand, plus tard, il lui succéda et finit par perdre son trône.

En conclusion, nous dirions que le récit, où le réel et le symbolique se croisent, a pour objectif de faire apparaitre les éléments qui déterminent l'identité généalogique de Démarate comme avantageuse par rapport à Cléomène, scellée par l'attribut royal de son père, grâce à la faveur divine triplement manifestée, ainsi que par le soupçon d'une filiation douteuse.

En somme, le récit se prête, par ses éléments, à une bonne interaction entre politique et littérature. Le serment dirige le déploiement de la narration, dont il constitue l'élément structurel ( $c f$. IV, 154; IX, 109), prépare la tromperie d'Ariston lui-même à travers le fantôme d'Astrabakos et révèle l'ironie dramatique $^{30}$, le roi qui recourt à l'élément surnaturel ${ }^{31}$ pour tromper finissant par être également trompé lui-même. La description des protagonistes laisse comprendre qu'ils agissent souvent malgré leur volonté : Ariston à cause de sa stérilité, sa troisième conjointe à cause de l'amour qu'elle a inspiré, Agétos à cause du serment trompeur - tous pris dans les filets de l'ảváxy nombre d'actions humaines ${ }^{32}$. Une lecture plus serrée révèle aussi des idées caractéristiques de l'anthropologie hérodotéenne: l'action du divin au niveau humain, ainsi que la participation et la responsabilité humaines, la compensation d'une faveur exceptionnelle du sort, ainsi que la tiøıs. Ces points, en

\footnotetext{
${ }^{28}$ BOEDEKER, l.c. (n. 24), p. 189, sans entrer dans les détails, reconnaît un « Helen pattern ».

29 Selon HARrison, ibid., p. 76, « Prayers answered... constitute proof of divine intervention » chez Hérodote.

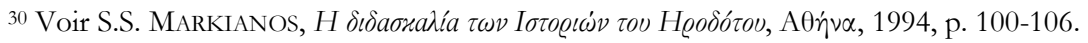

${ }^{31}$ L'aspect surnaturel du serment concerne sa liaison directe avec la $\theta \varepsilon o x \varrho \iota \sigma i \alpha$, les dieux qui garantissent son respect et punissent les transgresseurs: W. BURKERT, Griechische Religion der archaischen und klassischen Epoche, Stuttgart, 1977, p. 377-382.

32 Voir H. Schreckenberg, Ananke, Münich, 1964 (Zetemata, 36); P. Hohti, «Über die Notwendigkeit bei Herodot», Arctos 9 (1975), p. 31-37; G. LaChenaud, Mythologies, Religion et Philosophie de l'histoire dans Hérodote, Lille/Paris, 1978, p. 63-103; R. Vignolo Munson, "Ananke in Herodotus », JHS 121 (2001), p. 30-50.
} 
relation avec les expédients narratifs précédemment mentionnés, créent une espèce de mythe tragique: Ariston était sans enfants mais, en compensation et par sa propre initiative, a pris une épouse 'divine'; il montra de l'arrogance et de la ruse, qu'il paya par le doute concernant la paternité de son fils. Démarate, doté d'une origine 'divine', assume en compensation une paternité douteuse. L'oratio recta, en opposition avec l'oratio obliqua du récit antérieur, indique qu'Hérodote avait ses raisons d'approuver le récit considéré.

\subsubsection{Royauté}

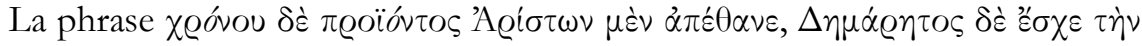
$\beta \alpha \sigma i \lambda \eta$ inv constitue l'épilogue de cette remontée vers le passé de Démarate via

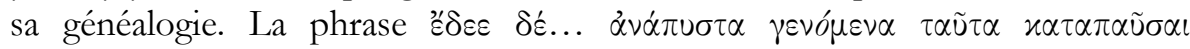

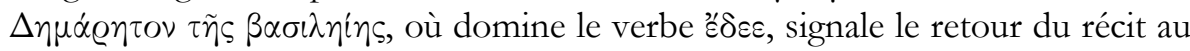
temps présent. Le verbe s'associe à la causalité métaphysique, qui renvoie à deux conceptions herodotéennes fondamentales: l'action du destin ${ }^{33}$ et le principe selon lequel le malheur est une rétribution pour une action antérieure sacrilège ou injuste ${ }^{34}$. La perte de la royauté apparait donc comme la conséquence inévitable d'une action irréfléchie, de son désaveu par son père, une conséquence cependant qu'a motivé l'attitude de Démarate lui-même contre

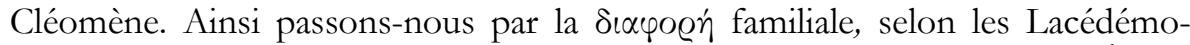
niens, à l'hostilité personnelle, due, selon Hérodote, aux événements d'Égine, mais aussi aux événements, plus anciens, ceux de Corinthe $(V, 75)$ qui ont comme conséquence le vote d'une loi interdisant le départ simultané des deux rois en vue d'une expédition, événement qui fonctionna au profit de Cléomène $^{35}$. Ce fait eut comme conséquence la supplantation de Démarate et la soumission, ensuite, de son rival qui essaya de se défaire d'un adversaire dérangeant ${ }^{36}$. À l'explication romanesque de l'hostilité familiale Hérodote oppose donc une explication réaliste : les raisons se réfèrent au présent, elles concernent les protagonistes personnellement et sont liées à des questions de pouvoir.

\subsubsection{Détrônement}

Afin d'écarter son rival du pouvoir, Cléomène a comploté avec le parent de son corrégnant, Leutychidès, en lui offrant des contreparties politiques. Cléomène exploite la vieille haine de Leutychidès contre Démarate, qu'Hérodote raconte par le moyen d'une analepse : bien que Leutychidès fût fiancé à la

\footnotetext{
33 Voir Lachenaud, o.c. (n. 32), p. 89-113; Harrison, o.c. (n. 17), p. 231 sur le passage cidessus ainsi que p. 223-242 sur l'action du destin chez Hérodote en général.

34 Voir Harrison, ibid., p. 105-106. Cf. LaChenaud, ibid., p. 99 et p. 93 sq. Pour la significa-

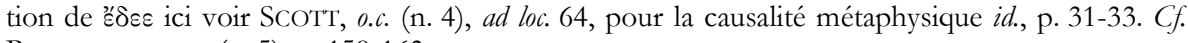
PERYSINAKIS, o.c. (n. 5), p. 159-163.

35 Voir LenSCHAU, l.c. (n. 25), p. 420; P. CARLIER, « La vie politique à Sparte sous le règne de

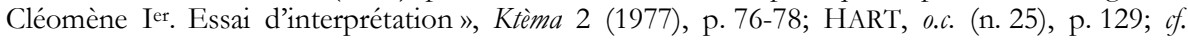
SCOTT, o.c. (n. 4), p. 214.

36 Voir Lenschau, supra, p. 425-426. Cf. HoOKer, l.c. (n. 16), p. 124.
} 
fille de Chilon, Démarate s'en est emparé avant qu'ils n'arrivent au mariage et l'a ensuite épousée ${ }^{37}$. La punition pour l'ancienne injustice est consommée, lorsque Leutychidès dénonce Démarate en contestant son droit d'être roi, parce qu'il n'était pas fils d'Ariston. Pour le prouver, il évoque le désaveu par son père devant les éphores, qui n'étaient pas d'accord entre eux sur ce sujet. Par conséquent, le recours à l'oracle de Delphes s'avère encore une fois nécessaire. Cette fois la réponse n'est plus ambiguë, la prophétesse que Cléomène s'est associée à temps ${ }^{38}$ ayant confirmé l'assertion de Leutychidès ${ }^{39}$.

Ce récit présente des ressemblances importantes avec le récit précédent. D'abord, les liens avec le mythe d'Hélène: Démarate est présenté comme revendiquant une femme qui ne lui appartient pas. Comme Pâris, il dissout une union légale et enlève la femme de son rival. Ainsi, Démarate, comme son père, Ariston, paraît agir d'une manière analogue à celle de Pâris. Les serments, qui précédemment l'ont favorisé, puisqu'ils ont conduit à l'enlèvement frauduleux de la mère 'divine', semblent cette fois lui nuire, car ils sont utilisés pour soulever à nouveau la question de la paternité contestée : lors du procès ${ }^{40}$, Leutychidès évoque la scène du refus d'Ariston de reconnaittre son fils, dans le but de créer un problème politique et d'en tirer un profit personnel. Les éphores réapparaissent dans le rôle de témoins oculaires, sans cependant intervenir effectivement. Dans les deux récits, c'est la généalogie qui jouera le rôle décisif, sans pour autant produire les mêmes effets : précédemment elle a confirmé le droit du roi au pouvoir, alors que maintenant elle est utilisée dans le sens contraire.

Même l'invocation de l'oracle conduit à des comparaisons du même ordre concernant la généalogie de la maison cette fois : l'origine de quelqu'un est mise en cause, sans qu'une solution puisse exister. Les Spartiates interviennent, le centre religieux est sollicité pour donner la solution, certains exploitent sa réponse pour acquérir des bénéfices politiques. Dans le texte, il est question d'un parallélisme entre le sort de Démarate et celui de Proclès : comme celui-ci, son descendant perd le pouvoir avec le 'sceau' de l'oracle. Les deux cas sont toutefois différents, car l'oracle n'œuvre plus pour l'équilibre, il participe à la machination dressée par Cléomène, qui exploita la possibilité offerte par son

${ }^{37}$ Selon MCQueEN, o.c. (n. 3), ad loc. 65, 2 : «Bride snatching was a well established custom at Sparta. » Cf. HoDKInson, Property and Wealth in Classical Sparta, London, 2000, p. 98. Une lecture différente par P. CARTLEDGE, «Spartan Wives: Liberation or Licence ?», CQ 31 (1981), p. 100. bribe ».

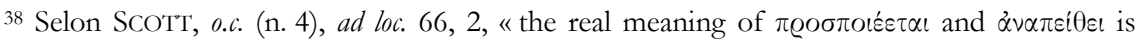

${ }^{39}$ Sur le complot à l'encontre de Démarate, avec la collaboration de Delphes, voir PARKE Wormell, o.c. (n. 12), p. 161. Cf. CRAHAY, o.c. (n. 13), p. 164; KIRCHBERG, o.c. (n. 12), p. 67; J.D. Mikalson, Herodotus and Religion in the Persian Wars, Chapel Hill/London, 2003, p. 118; DE STE Croix, o.c. (n. 24), p. 435.

40 Voir Macan, o.c. (n. 8), ad loc. 65, 10. Sur les procès des rois à Sparte et celui de Démarate voir H.W. PARKe, "The Deposing of Spartan Kings », CQ 39 (1945), p. 106-112; LÉvY, o.c. (n. 10), p.177-178. Une opinion différente est exprimée par A. LUTHER, Könige und Ephoren, Frankfurt am Main, 2004, p. 116. 
rang pour influencer Delphes afin d'obtenir la réponse souhaitée en arrivant à ses fins de manière frauduleuse ${ }^{41}$. Il paraît donc intervenir de nouveau dans la vie politique de Sparte en légitimant une machination politique, servant ainsi les finalités du pouvoir en place. Le moyen utilisé est encore une fois la généalogie.

Hérodote semble partager l'opinion selon laquelle celle-ci a été utilisée comme prétexte. Cela ressort du caractère subjectif attribué aux affirmations de Leutychidès ( $\varphi \alpha ́ s)$, mais surtout de l'accent mis sur la responsabilité de Cléo-

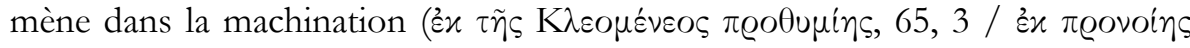

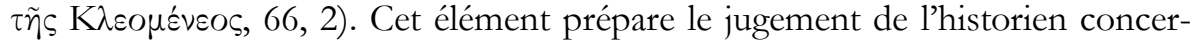
nant la punition et le décès honteux de celui-ci (VI, 84, 3). Enfin, Hérodote croit que les causes de la crise de l'institution étaient politiques et non mythi-

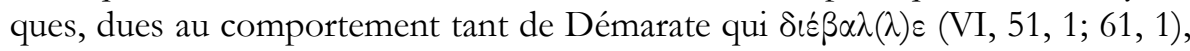
que de Cléomène qui n'a pas hésité à utiliser tous les moyens pour atteindre son rival. À cause du $\varphi \theta$ óvoৎ qui l'a conduit à la $\delta \iota \alpha \beta o \lambda \dot{n}$, Démarate connut le changement soudain de la fortune ${ }^{42}$ pour la même raison que celle à laquelle il devait sa faveur, à savoir son origine, et cela constitue une ironie tragique (ce qui évoque par certains aspects le cas d'(Edipe) : la paternité contestée était l'une des deux accusations habituelles qu'utilisaient les Spartiates pour neutraliser leur adversaire politique, l'autre étant l'accusation de subornation ${ }^{43}$.

Bref, la généalogie n'a pas été exploitée par les acteurs politiques de Sparte seulement, mais aussi par Hérodote, dans le but d'esquisser, malgré tout, un portrait sympathique de son héros, présenté comme un héros tragique, pris dans le filet des pouvoirs politique et religieux sans possibilité de réagir. Démarate est puni par la perte du pouvoir à cause d'un événement dont il n'était pas responsable. De ce point de vue, il fait penser à Crésus, qui perdit également son pouvoir à cause du meurtre commis par son ancêtre Gygès. Il y a entre ces deux hommes d'autres points communs : tous les deux connaissent le renversement soudain du sort, car ils sont victimes du p日óvos (dans le cas du roi lydien c'est la jalousie du divin, dans celui de Démarate c'est sa propre jalousie à l'égard de Cléomène); tous les deux ont une responsabilité personnelle dans leur mauvaise fortune. Dans les deux cas, il existe, d'une manière ou d'autre, une implication du divin et de l'oracle de Delphes dans leur destin. Enfin, les deux rois finissent au service d'un monarque perse. Les ressemblances vont jusqu'à la structure des deux récits, celui sur Crésus présentant des traits similaires, comme la narration exhaustive du mode d'accès au pouvoir, une brève

\footnotetext{
${ }^{41}$ Sur les relations de Cléomène avec Delphes voir CARTLEdge, o.c. (n. 4), p. 125 sq.

42 Sur ce sujet lié à l'idée de la marche cyclique des « choses humaines ", cf. D. LATEINER, The Historical Method of Herodotus, Toronto, 1989, p. 196 sq.; J. Romm, Herodotus, New Haven, 1998 ,

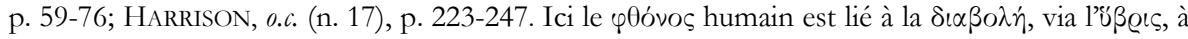
cause des $\pi \propto \varrho \varepsilon \delta ́ v \tau \omega \nu ~ \alpha َ \gamma \alpha \theta \tilde{\omega} \nu$ (voir III, 80, 3), semble-t-il, qui ont provoqué l'avidité de Démarate et sa punition. Sur la relation entre $\varphi \theta$ óvo et üßeıs, Perrsinakis, o.c. (n. 5), p. 169 sq.

${ }^{43}$ Voir S. LuRIA, "Zum politischen Kampf in Sparta gegen Ende des 5. Jahrhunderts », Klio 21 (1964), p. 404-420; LÉVY, o.c. (n. 10), p. 179.
} 
référence à la royauté, une emphase sur les circonstances de sa perte - encore un échantillon du modèle hérodotéen, bien connu, de l'ascension et de la chute d'un souverain ${ }^{44}$. Si nous avons raison, Démarate devait passer l'épreuve, afin d'accéder, comme Crésus, à une sagesse intérieure ${ }^{45}$, puisque nous le rencontrons plus tard à côté de Xerxès en tant que son sage conseiller ${ }^{46}$. Finalement, on peut poser la question de savoir si l'historien n'a pas exploité le thème généalogique pour créer le type du roi grec correspondant au roi lydien, en essayant de revaloriser le profil politique de Démarate.

\subsection{Le cheminement de Démarate après son éviction}

Il s'agit d'un récit, délibérément étendu pour justifier son parcours, constitué par trois parties: (1) sa querelle publique avec Leutychidès (VI, 67); (2) son origine (la version de sa mère: VI, 68-69); (3) sa fuite de Sparte vers la Perse (VI, 70, 1-2).

\subsubsection{Leutychidès et Démarate}

Les points importants sont : a) l'élection du roi déchu à une autre fonction montrant que l'action de son rival n'a pas rencontré l'approbation des Spartiates ${ }^{47}$; b) la raillerie publique de la part de Leutychidès, une offense à l'égard de sa $\tau \mu \dot{\eta}$, qui l'a incité d'une part à remettre en cause son existence ainsi que son cheminement ultérieur et de décider de clarifier la question de sa paternité; d'autre part, elle a lui offert une bonne justification pour sa fuite; c) sa réaction typiquement spartiate (réponse laconique, $\alpha i \delta \omega ́ s$ et silence ${ }^{48}$ ), qui vise à renforcer son prestige.

\subsubsection{Origine (la version de sa mère)}

Dans cette version, où se mêlent le niveau surnaturel et le niveau réel, dominait un $\varphi \alpha ́ \sigma \mu \alpha$, sous la forme d'Ariston, qui approcha la mère de Démarate, lui offrit des couronnes et dormit avec elle. Sa narration complique les choses, puisque celle-ci n'est pas en mesure de décider qui est son père, le héros Astrabakos ou Ariston ${ }^{49}$. Ainsi s'impose le thème de la double paternité. Une

\footnotetext{
${ }^{44}$ Voir H.R. ImMERwAHR, Form and Thought in Herodotus, Cleveland, Ohio, 1966, p. 76.

${ }^{45}$ Sur $\pi \dot{\alpha} \theta \varepsilon \iota \iota \dot{\alpha} \theta$ os chez Hérodote voir ImMERWAHR, supra, p. 85 n. 20; H.-P. STAHL, «Learning through Suffering? Croesus' Conversations in the History of Herodotus », YCS 34 (1975), p. 1-36; PeRYSINAKIS, o.c. (n. 5), p. 126 et n.1, 2.

${ }^{46}$ Voir H. Bischoff, Der Warner bei Herodot, Diss., Marburg, 1932, p. 68sq.; R. LATTimORE, "The Wise Adviser in Herodotus", CPh 34 (1939), p. 24-35; BoEDEKER, l.c. (n. 24), p. 191; PERYSINAKIS, o.c. (n. 5), p. 151 sq.

47 Voir SCOTT, o.c. (n. 4), ad loc. 67, 1. Cf. HART, o.c. (n. 25), p. 137.

48 Voir N. Richer, "Aidõs at Sparta» in Sparta. New Perspectives, p. 96-107; Cf. E. DAvid, «Sparta's kosmos of silence », ibid., p. 131.

49 Sur cet héros voir H. vON GEISAU, s.v. "Astrabakos », in Roscher's Lex. 1 (1964), col. 659; W. Burkert, «Demaratos, Astrabakos und Herakles. Königsmythos und Politik zur Zeit der Perserkriege (Herodot 6, 67-69)», MH 21-22 (1964-65), p. 175, A. SEEBERG, "ASTRABICA (Herodotus VI.68-69) », SO 41 (1966), p. 48-74; SCOTT, o.c. (n. 4), ad loc. 69, 1.
} 
telle union renvoie à des mythes grecs anciens connus, dont certains ont un rapport avec notre sujet. C'est une série de figures féminines mythiques, celles de Danaé, ancêtre des Héraclides, de la mère d'Hélène, 'protectrice' de la mère de Démarate, et, finalement, d'Alcmène, la mère d'Héraclès, ancêtre des rois de Sparte, autant de femmes avec lesquelles Zeus a couché en se présentant à elles sous les formes diverses: pluie, cygne, Amphitryon. Ce dernier mythe est le plus proche, faisant ressembler Démarate à Héraclès : comme celui-ci, le roi de Sparte parait avoir un père naturel, le héros Astrabakos, et un père adoptif, le roi Ariston.

Des quatre versions concernant son père (le mortel Agétos, le palefrenier d'Ariston, le roi, le héros), sa mère choisit de défendre les deux plus célèbres, car Astrabakos renforce les liens de son fils avec l'élément surnaturel, Ariston sa position politique. Ainsi, elle apparait dans un rôle équivalent à celui d'Argeia, un constat qui nous conduit à un autre symbolisme associant Démarate avec les ancêtres des deux maisons royales: comme Argeia, elle essaie de garantir les droits de son enfant en adoptant, même dans son argumentation, une attitude ambivalente concernant la question critique. Ariston a douté, parce qu'il ne savait pas que les femmes peuvent accoucher le neuvième mois, tout comme le septième. L'ambivalence facilite encore une fois les ambitions d'une mère dans un épisode montrant son fils favorisé à nouveau par des puissances surnaturelles, ce qui renvoie aussi à un mythe de tragédie.

Cette histoire romancée, avec un teint d'intensité dramatique, vise surtout à mettre en valeur la ressemblance du roi avec l'ancêtre de la dynastie, Héraclès; sa relation privilégiée avec l'élément surnaturel; ses liens avec ses antécédents royaux - en somme, tout ce qui fonde un droit héréditaire au trône supérieur à celui de Cléomène. Ainsi sommes nous d'accord avec ceux qui ont soutenu que l’origine de Démarate a été utilisée par des ennemis et des amis comme arme dans le champ de la confrontation politique ${ }^{50}$. L'importance de cette origine est prouvée par le fait que le récit, qui est axé sur la crise politique (VI, 61-69), commence et se termine avec la généalogie du roi. Hérodote n'est donc pas hors de propos dans sa présentation de cette affaire ${ }^{51}$.

\subsubsection{Le refuge en Perse}

La raillerie publique contre Démarate semble avoir marqué son cheminement, puisque les explications de sa mère n'ont pas été capables de le garder à Sparte. Son choix de se réfugier en Perse ${ }^{52}$ après son entretien dramatique avec elle aurait pu être lu en termes tragiques, c'est-à-dire comme un procès

\footnotetext{
${ }^{50}$ Voir Dovatour, l.c. (n. 24), p. 466-468; BurkerT, l.c. (n. 49), p. 175.

${ }^{51}$ Cf. St. DE VIDO, «Genealogie di Spartani re nelle Storie erodotee », QS 53-54 (2001-2002), p. 223 , n. 4.

52 Sur les raisons de sa fuite : D. Hereward, «The Flight of Demaratos », RhM 101 (1958), p. 238-249; RICHER, l.c. (n. 48), p. 107; LÉVY, o.c. (n. 10), p. 164; SCOTT, o.c. (n. 4), ad loc. 67, 3.
} 
expiatoire qui écrit la fin d'une aventure pour laquelle lui-même était aussi responsable. Ce qui étonne ici, c'est qu'aucun commentaire sur son action de se réfugier dans un pays hostile n'est mentionné, ni même à propos de l'accueil chaleureux qu'il a rencontré là-bas. Serait-ce un indice d'une assistance politique éventuelle de la part de ses compatriotes ou bien une omission délibérée d'Hérodote?

La longue référence à Démarate ne se termine pas avec sa fuite. Par des vaet-vient répétés entre passé et présent, Hérodote nous informe, dans les chapitres suivants (VI, 71-84), sur la fin déshonorante de ses ennemis. Pour expliquer leur mort il propose sa version de causalité surnaturelle, rejetant les

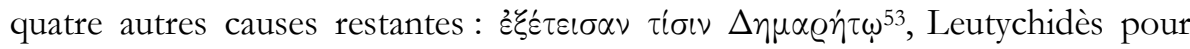
subornation, Cléomène pour la même raison et pour avoir incendié un bois sacré. Même dans sa justification, l'historien use de modèles tragiques.

\section{Conclusions}

À la fin de ce parcours, un examen général des données de l'enquête conduit aux conclusions suivantes: les généalogies des rois de Sparte ont constitué un domaine propice à l'exploitation politique en déterminant la vie politique de la cité, comme l'ont montré l'instauration de la double royauté et le détrônement de Démarate. En même temps, ces mêmes généalogies ont constitué, pour de nombreuses raisons, un thème particulièrement fécond pour Hérodote. D'abord pour des raisons politiques : une grande partie de la digression relative aux mauvais fonctionnements de la dyarchie à Sparte concerne la biographie de Démarate. La façon dont cette digression a été esquissée dévoile, croyons-nous, l'intention de l'historien de se concentrer sur la justification politique de celui-ci : petit à petit il progresse d'une narration qui concerne la primauté de Cléomène et que l'historien subvertit discrètement (généalogie familiale, VI, 51-55) vers la description des prérogatives égales des deux rois (VI, 56-60), pour déboucher sur une présentation de leurs relations interpersonnelles (généalogie individuelle, 61-70), donnant finalement plus de poids à Démarate. Notre lecture est renforcée par une série d'autres éléments : l'effort d'Hérodote de nous convaincre, dès le début, de l'équivalence des deux maisons royales, sa manière de présenter l'épreuve que le roi a subie quant à sa paternité, l'éloge qui clôt le récit le concernant, le fait que, ni ici ni ailleurs, il ne blâme Démarate pour trahison et l'interprétation qu'il choisit concernant la mort de ses rivaux, en assumant la responsabilité de son choix. Le thème de cette généalogie fatale est au centre de la stratégie narrative et permet à Hérodote de procéder implicitement à une comparaison entre les deux rois-

\footnotetext{
53 Voir VI, 72, 1; VI, 84, 3. Cf. VI, 74, 1 et 75, 3. Sur la relation du décès de Cléomène à la poursuite de Démarate, $c$. S. BENARDETE, Herodotean Inquiries, Hague, 1969, p. 164sq. Sur le sujet de la punition divine pour impiété, voir MikAlson, o.c. (n. 39), p. 142 sq. Cf. W. Desmond, «Punishment and the Conclusion of Herodotus' Histories», GRBS 44 (2004), p. 19-40.
} 
ennemis : Démarate est le représentant le plus authentique de la tradition spartiate, puisque toutes les périodes de sa vie sont soumises à des symboles spartiates fondateurs : Dioscures, Helène, Astrabakos, Héraclès, les chefs de la famille royale. De plus, il fonde un droit héréditaire sur le pouvoir, supérieur à celui de Cléomène parce que, en dehors du droit héréditaire à la royauté, il porte, en quelque sorte, un 'sceau' divin tant par les interventions surnaturelles

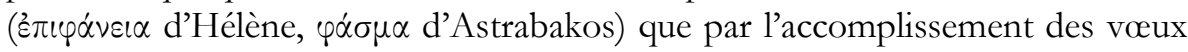
du peuple (Démarate $<\delta \tilde{\eta} \mu o \varsigma+\dot{\alpha} \varrho \tilde{\omega} \mu \alpha l)$ priant pour sa naissance. Dans la personne de Démarate semblent converger les pouvoirs religieux et politique.

Bien que certaines conjectures aient été faites ${ }^{54}$, il demeure difficile de deviner les raisons de la sympathie d'Hérodote à l'égard de Démarate, malgré les défauts de celui-ci. Nous pouvons seulement dire que cette sympathie s'avère fonctionnelle sur le plan narratif, car elle annonce le rôle de Démarate, sage conseiller, et justifie la mort horrible de son corrégnant.

Par conséquent, à côté des raisons politiques, Hérodote avait aussi des raisons narratives de s'intéresser aux généalogies. Du point de vue de la structure, en les choisissant comme trame de son récit, il associe le passé au présent par un lien causal, en obtenant la cohérence narrative souhaitée. En plus, à travers les biographies qui se créent, l'historien peut exposer en même temps l'histoire politique de Sparte en présentant les personnes qui l'ont déterminée. Du point de vue littéraire, la généalogie offre des éléments permettant une structure littéraire tragique implicite, qui suggère le côté dramatique du cheminement de Démarate en tant que victime d'une conspiration, rendant ainsi possible la compassion à son égard. Du point de vue de la technique narrative, les généalogies lui donnent l'occasion d'utiliser une variété de moyens narratifs qui servent les buts du récit et créent tension et suspense : l'analepse, le doublet (la répétition de la scène du désaveu), les 'reflets' de narration ${ }^{55}$ (comportement érotique d'Ariston, de Pâris / de Démarate; destin de Proclès / de Démarate), ainsi que le conflit/contraste qui parcourt toute la description.

Enfin, Hérodote profite des généalogies pour mettre en valeur l'aspect moral de l'action, la responsabilité humaine dans l'évolution des événements, formulant ainsi des idées fondamentales dans son œuvre : l'idée des conséquences funestes de la calomnie, la marche cyclique de l'histoire, la punition divine, autant d'idées qui donnent à son œuvre une place particulière dans l'historiographie grecque.

Gerasimoula ZOGRAPHOU

Université de Jannina

Institut de Philologie Classique

GR - 45110 JANNINA

54 Voir par ex. S. ForsDyKe, "Greek History c.525-480 BC», in E. BAKKER, I. DE JONG, H. vAN WEES (éds), Brill's Companion to Herodotus, Leiden, 2002, p. 546.

${ }^{55} \mathrm{Il}$ s'agit de faits qui se répètent, quant à la forme générale, mais avec d'autres protagonistes. 